\title{
On planarity of compact, locally connected, metric spaces
}

\author{
R. Bruce Richter, Brendan Rooney \\ University of Waterloo \\ Carsten Thomassen \\ Technical University of Denmark
}

[ATEX-ed: November 18, 2008

\begin{abstract}
Thomassen [Combinatorica 24 (2004), 699-718] proved that a 2-connected, compact, locally connected metric space is homeomorphic to a subset of the sphere if and only if it does not contain $K_{5}$ or $K_{3,3}$. The "thumbtack space" consisting of a disc plus an arc attaching just at the centre of the disc shows the assumption of 2-connectedness cannot be dropped. In this work, we introduce "generalized thumbtacks" and show that a compact, locally connected metric space is homeomorphic to a subset of the sphere if and only if it does not contain $K_{5}, K_{3,3}$, or any generalized thumbtack, or the disjoint union of a sphere and a point.
\end{abstract}

\section{Introduction}

It is well-known that planarity of finite graphs was characterized in 1930 by Kuratowski [1], who showed that a finite graph is planar if and only if it does not contain a homeomorph of either the complete graph $K_{5}$ or the complete bipartite graph $K_{3,3}$. It is natural to wonder how this might generalize. Thomassen [3] proved the following.

Theorem 1.1 Let $X$ be a compact, locally connected, 2-connected metric space. Then $X$ is homeomorphic to a subspace of the sphere if and only if it does not contain a homeomorph of either $K_{5}$ or $K_{3,3}$.

The thumbtack space consists of a closed unit disc in the plane, together with a line segment, one end of which is the centre of the disc. It is a standard fact (and an easy consequence of Lemma 2.1 below) that the thumbtack space is not homeomorphic to a subspace of any 2-manifold and therefore, in particular, is not a subspace of the sphere. Thus, the assumption of $2-$ connection in Theorem 1.1 is required. 
In this work, we completely characterize planarity for compact, locally connected metric spaces. Such a space has only finitely many components. If none of them is a sphere, then the whole space embeds in the sphere if and only if each component embeds in the sphere. (While we are on this small point, we use the word planar to mean is homeomorphic to a subspace of the sphere. Except for the sphere itself, this is the same as being homeomorphic to a subspace of the plane.) Thus, it will suffice to work with connected spaces. In the next section, we describe a general version of the thumbtack space and in the following two sections we shall prove our main result:

Theorem 1.2 A compact, locally connected metric space $X$ is not planar if and only if $X$ contains either $K_{5}$ or $K_{3,3}$ or a generalized thumbtack or the disjoint union of a sphere and a single point.

If $X$ contains one of the non-planar spaces listed in the theorem, then $X$ is not planar. The interesting point is the converse.

\section{Webs}

In [3] Thomassen suggested that Theorem 1.1 extends to connected, locally connected compact metric spaces if we also exclude certain subspaces of the thumbtack space. However, the description given in [3] does not suffice. In this section we introduce the generalization of the thumbtack space that is the remaining forbidden structure for planarity.

Let $X$ be a topological space and let $Y$ be a subspace of $X$. A $Y$-bridge in $X$ is the closure $B$ of a component of $X \backslash Y$. The attachments of $B$ are the elements of $B \cap Y$.

In the cases of interest, $X$ is a locally connected space and $Y$ is a closed subset of $X$. In the important case that $Y$ is a homemorph of a circle, the attachments of a $Y$-bridge $B$ naturally occur in the cyclic order determined by $Y$. A residual arc of $B$ is the closure in $Y$ of a component of $Y \backslash B$. In particular, every point of $Y \backslash B$ is in a unique residual $\operatorname{arc}$ of $B$.

If $B$ and $B^{\prime}$ are distinct $Y$-bridges and $Y$ is again a homeomorph of a circle, then either all the attachments of $B$ are in the same residual arc of $B^{\prime}$ or they are not. In the latter case, $B$ and $B^{\prime}$ overlap. It is not hard to see that this is a symmetric condition, that is, if all the attachments of $B$ are in the same residual arc of $B^{\prime}$, then all the attachments of $B^{\prime}$ are in the same residual arc of $B$.

It is also not hard to see that $B$ and $B^{\prime}$ overlap if and only if either there are attachments $x, y$ of $B$ and $x^{\prime}, y^{\prime}$ of $B^{\prime}$ so that $x, x^{\prime}, y, y^{\prime}$ are distinct and occur in this cyclic order in $Y$ - in this case $B$ and $B^{\prime}$ are skew, or $B$ and $B^{\prime}$ both have just three attachments and they are all the same - in this case $B$ and $B^{\prime}$ are 3 -equivalent. (Here is an argument slightly easier than the one given in [3]. If one of them, say $B$, has an attachment $x$ that 
is not an attachment of $B^{\prime}$, then pick $x^{\prime}$ and $y^{\prime}$ to be the ends of the residual arc $A$ of $B^{\prime}$ containing $x$. Since not every attachment of $B$ is in $A$, there is an attachment $y$ of $B$ in $Y \backslash A$ and, therefore, the vertices $x, x^{\prime}, y, y^{\prime}$ are distinct and occur in this cyclic order in $Y$. Otherwise the attachments of $B$ and $B^{\prime}$ are identical. If there are not at least three attachments, then they do not overlap. If there are at least four attachments, then any four may be chosen as $x, x^{\prime}, y, y^{\prime}$.)

As an historical remark, we note that Tutte developed the theory of bridges (including the notion of overlapping bridges) in graphs and binary matroids in the course of several papers. It is difficult to cite references for particular results in the subject. Bridges appear in [5] under the name $J$-components and also in [6]. The book [7] is a good reference for material about bridges.

A web with centre $w$ in a locally connected metric space $X$ is a closed connected subspace $W$ of $X$ in which there is a sequence $\left(C_{i}\right)_{i \geq 0}$ of disjoint circles with the following two properties:

1. for each $i>0$, there are two overlapping $C_{i}$-bridges in $W$, one containing $C_{0}, C_{1}, \ldots$, $C_{i-1}$, and the other containing $C_{i+1}, C_{i+2}, \ldots$;

2. every neighbourhood of $w$ contains all but finitely many of the $C_{i}$.

A generalized thumbtack consists of a web $W$ with centre $w$ together with a line segment, one end of which is $w$. Moreover, the line and the web are internally disjoint; that is, they have only $w$ in common. If $W$ is a web with centre $w$ embedded in the sphere, then each of the $C_{i}(i>0)$ has the property that the two $C_{i}$-bridges in $W$ are embedded in different faces of $C_{i}$. Thus, if $W$ is the web of a generalized thumbtack, there is no face of $W$ incident with $w$. This proves the following.

Lemma 2.1 No generalized thumbtack embeds in the sphere.

\section{Web-free blocks}

Our main result is that if $X$ is a locally connected, compact metric space that has no $K_{5}, K_{3,3}$, generalized thumbtack or the disjoint union of a sphere and a point, then $X$ is homeomorphic to a subspace of the sphere. By earlier remarks, it suffices to consider the case $X$ is connected. In view of Theorem 1.1, we may further assume that $X$ is not 2-connected, so there is at least one point $x$ of $X$ for which $X \backslash\{x\}$ is not connected. Such an $x$ is a cut point of $X$.

If $K$ and $L$ are subspaces of $X$ with no cut points and $|K \cap L| \geq 2$, then $K \cup L$ also has no cut points. Thus, it is an easy application of Zorn's Lemma to see that $X$ has maximal connected subspaces with no cut points; we shall call such a maximal subspace 
a block of $X$. The blocks with more than one point are precisely Whyburn's $E_{0}$-sets [8]. Obviously, distinct blocks intersect in at most one point, and a block is easily seen to be closed. A block is non-degenerate if it has more than one point (which is easily equivalent to saying it contains a simple closed curve).

The following lemma is the first of the two main points of the proof.

Lemma 3.1 Suppose $X$ is a connected, locally connected, compact metric space and that $B$ is a non-degenerate block of $X$ embeddable in the sphere. Then either $B$ has an embedding in the sphere so that each cut point of $X$ in $B$ is incident with a face of $B$, or $B$ contains a web centred at some cut point.

The second main point of the proof, which is in the next section, is to use this lemma to show that if every block of $X$ embeds in the sphere and $X$ has no generalized thumbtack, then $X$ embeds in the sphere. In order to prove Lemma 3.1, we need the following.

Lemma 3.2 Suppose $X$ is a connected, locally connected, compact metric space and $B$ is a block of $X$. Then $B$ contains only countably many points that are cut points of $X$.

Proof. Let $C$ denote the set of cut points of $X$ in $B$. For each $v \in C$, let $K(v)$ denote any component of $X-v$ not containing $B-v$.

The result is an immediate consequence of showing the following: for each positive integer $k$, the number of $v \in C$ for which the distance from $v$ to some point of $K(v)$ is at least $1 / k$ is finite.

Otherwise, there is a sequence $\left(v_{n}\right)$ of distinct points in $C$ and points $w_{n}$ in $K\left(v_{n}\right)$ so that the sequences $\left(v_{n}\right)$ and $\left(w_{n}\right)$ converge to $v$ and $w$, respectively, and, for all $n$, $\operatorname{dist}_{X}\left(v_{n}, w_{n}\right) \geq 1 / k$. Then $\operatorname{dist}_{X}(v, w) \geq 1 / k$ as well. Let $V$ and $W$ be disjoint, connected open neighbourhoods of $v$ and $w$, respectively. For $n$ sufficiently large, $v_{n}, v_{n+1} \in V$ and $w_{n}, w_{n+1} \in W$.

Let $A$ be an arc in $W$ joining $w_{n}$ and $w_{n+1}$. Obviously $A$ is disjoint from $\left\{v_{n}, v_{n+1}\right\}$, a contradiction, since every $w_{n} w_{n+1}-\operatorname{arc}$ must contain both $v_{n}$ and $v_{n+1}$.

The remainder of this section is devoted to proving the following, which clearly combines with Lemma 3.2 to prove Lemma 3.1.

Lemma 3.3 Suppose $X$ is a 2-connected, locally connected, compact metric space embeddable in the sphere and $S$ is a countable subset of $X$. Then either $X$ has an embedding in the sphere so that each point of $S$ is incident with a face of $X$, or $X$ contains a web centred at some point of $S$.

Proof. The following claim is central to the proof. 
Claim 1 Let $w$ be an arbitrary point of $X$ and suppose $X$ has no web centred at $w$. Then there is a point $w^{\prime} \in X \backslash\{w\}$ so that, if we add an arc $A$ joining $w$ and $w^{\prime}$ that is internally disjoint from $X$, then $X \cup A$ embeds in the sphere. In particular, any embedding of $X \cup A$ in the sphere induces an embedding of $X$ in the sphere having $w$ incident with a face.

Proof. Suppose we have a connected subspace $W_{i}$ of $X$ containing simple closed curves $C_{1}, \ldots, C_{i}$ so that, for each $j$ with $1<j \leq i$, there are two overlapping $C_{j}$-bridges, one containing $C_{1} \cup \cdots \cup C_{j-1}$, and the other containing $C_{j+1} \cup \cdots \cup C_{i} \cup\{w\}$. We claim that either we can extend the sequence to $C_{i+1}$, with $C_{i+1}$ within distance $1 / i$ of $w$ or we can find $w^{\prime}$ and introduce $A$.

Assume now that $X$ is embedded in the sphere. Consider a closed connected neighbourhood $N$ of $w$ having diameter (in $X$ ) less than $1 / i$ and less than the distance from $w$ to $C_{i}$. From [3, Cor. 4.4], either there is an arc in the sphere that is internally disjoint from $X$ and joins $w$ to some point of the face of $N$ containing $C_{i}$ or $N$ contains a simple closed curve $C$ separating $w$ from $C_{i}$ in the sphere. Since the former implies $w$ is incident with a face of $X$, we may assume it is the latter that occurs.

Now $C$ is disjoint from $C_{i}$, and the face $F$ of $C$ containing $w$ is different from the face of $C$ containing $C_{i}$. Let $M$ be the $C$-bridge containing $w$ and let $L$ be the union of the remaining $C$-bridges contained in $F$. Then $C \cup L$ is a $2-$ connected space and so by [2] the face $F^{\prime}$ of $C \cup L$ containing $M$ is bounded by a simple closed curve $C^{\prime}$ and there is only one $C^{\prime}$-bridge contained in $F^{\prime}$, namely $M$.

Let $w^{\prime}$ be an attachment of the $C^{\prime}$-bridge containing $C_{i}$. Consider the space $M^{+}=$ $M \cup C^{\prime} \cup \alpha$, where $\alpha$ is an arc internally disjoint from $X$ joining $w$ and $w^{\prime}$. If $M^{+}$is planar, then, in any planar embedding, $C^{\prime}$ bounds a face (since $M^{+} \backslash C^{\prime}$ is connected) and, therefore, the arc $\alpha$ may be added to $X$, as required. If it is not planar, then Theorem 1.1 implies it has a homeomorph $K$ of either $K_{5}$ or $K_{3,3}$. Since $M \cup C^{\prime}$ is planar, $\alpha \subseteq K$ and, therefore, $M \cup C^{\prime}$ has a simple closed curve $C_{i+1}$ so that the $C_{i+1}$-bridges containing $w$ and $w^{\prime}$ overlap. In particular, $w^{\prime} \notin C_{i+1}$ and so the $C_{i+1}$-bridge containing $w^{\prime}$ is the same as the one containing $C_{i}$, and overlaps the $C_{i+1}$-bridge containing $w$, as required.

We can enumerate the elements of $S$ as $a_{1}, a_{2}, \ldots$ We add to $X$ a sequence $\left(A_{i}\right)$ of arcs. If we have added $A_{1}, \ldots, A_{i-1}$ and $i^{\prime}$ is least such that $a_{i^{\prime}} \notin \cup_{j<i} A_{j}$, Claim 1 implies we can add an $\operatorname{arc} A_{i}$ such that: $a_{i^{\prime}}$ is an end of $A_{i}$; $A_{i}$ has diameter $<1 / i ; A_{i}$ is internally disjoint from $X ; A_{i}$ is totally disjoint from $\bigcup_{j<i} A_{j}$; and $X \cup\left(\bigcup_{j \leq i} A_{j}\right)$ is planar.

Claim $2 X \cup\left(\cup_{j \geq 1} A_{j}\right)$ is planar.

Proof. Notice that $X \cup\left(\bigcup_{j \geq 1} A_{j}\right)$ is a compact, 2-connected, locally connected metric space, so Theorem 1.1 implies that either $X \cup\left(\bigcup_{j \geq 1} A_{j}\right)$ is planar or it contains a subspace 
$K$ homeomorphic to either $K_{5}$ or $K_{3,3}$. Notice that $K$ has only 5 or 6 branchpoints $v_{i}$; these are points of $X$, so they have disjoint connected (in $X$ ) neighbourhoods $U_{i}$.

Consider the three or four branches of $K$ having $v_{i}$ as an end point. If possible, for each such branch $\beta$, let $b_{\beta, i}$ be a point of $\left(\beta \cap U_{i}\right)-v_{i}$. Otherwise, there is an $A_{j}$ contained in $\beta$ and containing $v_{i}$. In this case, let $b_{\beta, i}$ denote the other end of $A_{j}$. Let $\mathfrak{A}_{i}$ denote the union of such $A_{j}$. (It is also possible that $\beta$ is equal to some $A_{j}$; such an $A_{j}$ will be in $\mathfrak{A}_{i}$ for both its ends.)

For each branch $\beta$ of $K$, with ends $v_{i}$ and $v_{i^{\prime}}$, let $\beta^{\prime}$ be the subarc of $\beta$ joining $b_{\beta, i}$ and $b_{\beta, i^{\prime}}$. Every point $x$ of $X$ in $\beta^{\prime}$ has a connected neighbourhood $V_{x}$ in $X$, which may be chosen so that, if $x, x^{\prime}$ are in distinct $\beta^{\prime}$, then $V_{x}$ and $V_{x^{\prime}}$ are disjoint. Since $\beta^{\prime}$ is compact, $X \cap \beta^{\prime}$ is covered by finitely many of the $V_{x}$. Together with finitely many of the arcs $A_{j}$, the union of these finitely many $V_{x}$ provides a connected subspace of $X \cup\left(\cup_{j \geq 1} A_{j}\right)$ containing the ends of $\beta^{\prime}$. This subspace contains an arc $\beta^{\prime \prime}$ joining the ends of $\beta^{\prime}$, and $\beta^{\prime \prime}$ contains only finitely many of the $\operatorname{arcs} A_{j}$. Note the $\operatorname{arcs} \beta^{\prime \prime}$ are pairwise disjoint.

In $U_{i}$ there is a tree $T_{i}$ contained in $X$ and containing the $b_{\beta, i}$ that are in $U_{i}$. The space $L$ consisting of all the $T_{i}$, all the $\mathfrak{A}_{i}$, and all the $\beta^{\prime \prime}$ is a subspace of $X \cup\left(\bigcup_{j \leq J} A_{j}\right)$, for some positive integer $J$, and, therefore, embeds in the sphere. However, $L$ contains either $K_{5}$ or $K_{3,3}$, a contradiction. Hence $X \cup\left(\bigcup_{j \geq 1} A_{j}\right)$ is planar.

The proof of Lemma 3.3 is completed by observing that any embedding of $X \cup$ $\left(\bigcup_{j \geq 1} A_{j}\right)$ in the sphere provides an embedding of $X$ in the sphere so that every point of $S$ is incident with a face of $X$.

\section{Proof of Theorem 1.2}

We are now prepared to prove our main theorem. We start with the following.

Claim 1 For each positive integer n, there are only finitely many non-degenerate blocks with diameter at least $1 / n$.

Proof. Otherwise, there is an infinite sequence $\left(B_{i}\right)_{i \geq 0}$ of distinct blocks of $X$ all having diameter at least $1 / n$, for some positive integer $n$. Each $B_{i}$ contains two points $v_{i}$ and $w_{i}$ at distance $1 / n$, and the $B_{i}, v_{i}$ and $w_{i}$ may be chosen so that the sequences $\left(v_{i}\right)$ and $\left(w_{i}\right)$ both converge, say to $v$ and $w$.

There exist disjoint connected neighbourhoods $V$ and $W$ of $v$ and $w$, respectively. For $i$ sufficiently large, $v_{i}, v_{i+1} \in V$ and $w_{i}, w_{i+1} \in W$. There are disjoint arcs, one in $V$ joining $v_{i}$ and $v_{i+1}$ and one in $W$ joining $w_{i}$ and $w_{i+1}$. But this is impossible, since any $v_{i} v_{i+1}$-arc and any $w_{i} w_{i+1}$-arc must have a cut-point of $X$ in common. 
Let $B_{1}, B_{2}, \ldots$ be an enumeration of the non-degenerate blocks of $X$ and let $Y$ be a countable dense subset of $X$. Let $Y^{\prime}=Y \backslash \operatorname{cl}\left(\bigcup_{i} B_{i}\right)$. We note that $X=\operatorname{cl}\left(\left(Y^{\prime} \cup\left(\bigcup_{i} B_{i}\right)\right)\right.$. Enumerate $Y^{\prime}$ as $y_{1}, y_{2}, \ldots$ and now consider the sequence $B_{1}, y_{1}, B_{2}, y_{2}, \ldots$ We begin with $H_{0}$ being an embedding of $B_{1}$ so that every cut point of $X$ in $B_{1}$ is incident with a face of $H_{0}$.

After a given iteration, we have an embedding in the sphere of a connected, compact subspace $H_{i}$ of $X$ so that every cut point of $X$ in $H_{i}$ is incident with a face of $H_{i}$ and, if some $B_{j}$ has more than one point in $H_{i}$, then $B_{j} \subseteq H_{i}$. We now explain how to obtain $H_{i+1}$.

Consider the first term of the sequence $B_{1}, y_{1}, B_{2}, y_{2}, \ldots$ that is not in $H_{i}$. We will describe how to proceed assuming this term is a $B_{j}$; the same description applies to the case this term is a $y_{j}$.

Let $A$ be an arc in $X$ joining $B_{j}$ to $H_{i}$ that is internally disjoint from $H_{i} \cup B_{j}$ with ends $a \in H_{i}$ and $b \in B_{j}$. Let $\mathcal{B}$ denote the set of non-degenerate blocks that intersect $A$ in more than one point. For each $B \in \mathcal{B}, B \cap A$ is an arc with ends $a_{B}$ and $b_{B}$. Let $\alpha_{B}$ be a new arc internally disjoint from $X$ with ends $a_{B}$ and $b_{B}$. Let $\mathcal{B}^{\prime}$ denote the set of $B \in \mathcal{B}$ for which $B \cup \alpha_{B}$ is not planar.

Claim $2 \mathcal{B}^{\prime}$ is finite.

Proof. Otherwise, there is an infinite sequence $\left(B_{k}\right)$ of elements of $\mathcal{B}$ so that the ends $a_{k}, b_{k}$ of $B_{k} \cap A$ are strictly monotonic in $A$, that is, they are distinct and they occur in the order $a_{1}, b_{1}, a_{2}, b_{2}, \ldots$ in $A$. Because $A$ is an interval, these points converge to some $w$ in $A$. We claim that $X$ has a web centred at $w$.

For each $i$, Theorem 1.1 implies that $B_{i} \cup \alpha_{B_{i}}$ contains a subspace $K$ that is a homeomorph of either $K_{5}$ or $K_{3,3}$. Then $\alpha_{B_{i}} \subseteq K$, and $K-\alpha_{B_{i}}$ contains a simple closed curve $C_{i}$ so that $a_{i}$ and $b_{i}$ are in distinct, overlapping $C_{i}$-bridges in $K$. Thus, the sequence $\left(C_{i}\right)_{i \geq 1}$ is the sequence of simple closed curves of a web $W$ with centre $w$ (from Claim 1, the diameters of the $C_{i}$ tend to 0 ). If $w$ is not equal to $y_{j}$, then $W \cup A \cup H_{i} \cup B_{j}$ contains a generalized thumbtack, a contradiction. On the other hand, if $w=y_{j}$, then $y_{j}$ is in the closure of the union of the non-degenerate blocks (namely those in $\mathcal{B}^{\prime}$ used to constuct the web), which contradicts the definition of $Y^{\prime}$.

As we traverse $A$ from $H_{i}$ to $B_{j}$, we encounter the elements of $\mathcal{B}^{\prime}$ in the order $B_{1}^{\prime}, B_{2}^{\prime}, \ldots, B_{k}^{\prime}$. Reorder our sequence so that these blocks move in front of $B_{j}$ in the ordering $B_{1}, y_{1}, B_{2}, y_{2}, \ldots$. Then, instead of trying to introduce $B_{j}$, we will first introduce $B_{1}^{\prime}$, then $B_{2}^{\prime}, \ldots, B_{k}^{\prime}$ and finally $B_{j}$. These will be the steps we take to introduce $B_{j}$.

The point is that now, in order to introduce the next term in our enumeration, we may assume that every block $B$ in $\mathcal{B}$ has an embedding of $B \cup \alpha_{B}$ in the unit disc so 
that $\alpha_{B}$ is in the boundary of the disc. Set $B^{*}=A \cup \bigcup_{B \in \mathcal{B}} B$ and let $\alpha$ be a new arc internally disjoint from $B^{*}$ joining $a$ and $b$. Then $B^{*} \cup \alpha$ is 2-connected and planar (it is easily seen that it contains neither $K_{5}$ nor $K_{3,3}$ ) and so has an embedding in the sphere in which every cut point of $X$ in $B^{*}$ is incident with a face. We use this embedding to extend $H_{i}$ to $H_{i+1}$.

Let $F$ be a face of $H_{i}$ incident with $a$ and let $\gamma_{i}$ be a simple closed curve bounding a closed disc $\Delta_{i}$ contained in $F \cup\{a\}$. We embed $B^{*} \cup B_{j}$ in $\Delta_{i}$ by embedding $B^{*} \cup \alpha$ in the closed unit disc so that $a$ corresponds to $(-1,0), b$ corresponds to the origin, and $B^{*} \cup \alpha$ is contained inside the circle with radius $1 / 2$ and centre at $\left(-\frac{1}{2}, 0\right)$; likewise we embed $B_{j}$ in the circle with radius $1 / 4$ and centre at $\left(\frac{1}{4}, 0\right)$. We then homeomorphically transfer this embedding into $\Delta_{i}$.

The only other points to make about $\gamma_{i}$ and $\Delta_{i}$ are that: for $k<i$, either $\Delta_{i} \subseteq \Delta_{k}$ or $\Delta_{i} \cap \Delta_{k}=\emptyset$; the diameters of the $\Delta_{i}$ go to 0 ; if, for several $i$, we use the same point $y$ to attach the new block to $H_{i}$, we choose (for $k<i$ ) $\Delta_{i} \subseteq \Delta_{k}$; and, $\gamma_{i}$ and $\gamma_{k}$ should be disjoint except for such a $y$.

Now let $H^{*}$ denote $\bigcup_{i \geq 1} H_{i}$, so that $H^{*}$ is a connected subset of the sphere. We shall prove that the closure of $H^{*}$ is homeomorphic to $X$.

Claim 3 If $z$ is a cut point of $X$, then $z \in H^{*}$.

Proof. As $X \backslash\{z\}$ is not connected, it partitions into two open sets, both of which are open in $X$. Thus, each of these open sets has a point in $Y^{\prime} \cup \bigcup_{i} B_{i}$ and, therefore, some $H_{i}$ has a point in each of these open sets. These two points are connected by an arc in $H_{i}$ and this arc contains $z$.

Henceforth, we assume that $X \neq H^{*}$. By the claim, the only points of $X$ that are not contained in $H^{*}$ are non-cut points of $X$ not in any non-degenerate block. Therefore, every non-degenerate block has a cut point and the diameters of these blocks converge to 0 , so every $x \in X \backslash H^{*}$ is necessarily the limit of a sequence $\left(x_{i}\right)_{i \geq 0}$ of cut points $x_{i}$. We may choose the sequence to be monotonic, in the sense that they occur in this order in any arc joining $x_{0}$ to $x$.

It follows that, for each $i, x_{i+1}, x_{i+2}, \ldots$ are all contained in the same $x_{i}$-bridge $L_{i}$. Since $x$ is never added to any $H_{j}$, we know that, for each $j$, only finitely many $x_{i}$ are contained in $H_{j}$ (because $H_{j}$ is closed). When $x_{i}$ is added, it is inside some $\Delta_{j_{1}}$, and so all of $x_{i+1}, x_{i+2}, \ldots$ will be put inside $\Delta_{j_{1}}$. Thus, there is a nested sequence $\Delta_{j_{k}}$ of disjoint closed discs, each containing a tail of the sequence $\left(x_{i}\right)$. Since the diameters of the $\Delta_{j_{k}}$ are converging to 0 , there is a unique point in $\bigcap_{k} \Delta_{j_{k}}$; this is the point to which we map $x$.

Notice that every point in $H^{*}$ is in some $H_{j}$ and, therefore, is in the interior of only finitely many of the $\Delta_{i}$ 's. In particular, $x$ is not mapped onto any point of $H^{*}$. 
Furthermore, the argument given shows that this point in the sphere is independent of the particular sequence $\left(x_{i}\right)$.

In order to conclude the proof, we must show that we now have an embedding of $X$ in the sphere. Suppose $z$ and $z^{\prime}$ are distinct points of $X \backslash H^{*}$ and that $A$ is an arc in $X$ joining them. Every point of $A \backslash\left\{z, z^{\prime}\right\}$ is either a cut point of $X$ separating $z$ and $z^{\prime}$ in $X$ or is in a non-degenerate block of $X$. Since $z$ and $z^{\prime}$ are neither cut points nor in non-degenerate blocks, there are cut points of $X$ in $A$. In particular, $z$ and $z^{\prime}$ are mapped to distinct points of the sphere. It follows that our map is one-to-one.

To prove continuity, it will be a help to distinguish the original space $X$ and its elements from their images in the sphere. For their images in the sphere, we shall place 's on the symbols, as in $X^{\prime}, H_{i}^{\prime}$ and so on. Let $\left(z_{n}\right)$ be a sequence in $X$ converging to $z$. We shall prove $\left(z_{n}^{\prime}\right)$ converges to $z^{\prime}$. We distinguish two cases, depending on $z$.

Case 1. $z$ is not in $H^{*}$.

In this case, $z^{\prime}$ is the unique point in $\bigcap \Delta_{j_{i}}$, for some nested sequence $\left(\Delta_{j_{i}}\right)$. Let $y_{i}^{\prime}$ be the point of $\gamma_{j_{i}} \cap H^{* \prime}$. Obviously all but finitely many of the $z_{n}$ are in the same component of $X \backslash\left\{y_{i}\right\}$ as $z$, and therefore all but finitely many of the $z_{n}^{\prime}$ will appear in $\Delta_{j_{i}}$. Since this is true for all $i,\left(z_{n}^{\prime}\right)$ converges to $z^{\prime}$, as required.

Case 2. $z$ is in $H^{*}$.

Let $\varepsilon>0$ be given. Let $k_{0}<k_{1}<\cdots$ be the indices $k$ so that $\gamma_{k}$ contains $z^{\prime}$. If this sequence is finite, then let $k^{*}$ be the largest term in the sequence (or $k^{*}=0$ if there is no such $k_{i}$ ). If this sequence is infinite, then let $k^{*}$ be any one of these for which $\Delta_{k_{i}}$ has diameter less than $\varepsilon$. At the moment $\Delta_{k^{*}}$ is created, it is to extend $H_{k^{*}-1}$ to $H_{k^{*}}$.

Partition the positive integers into the two sets $N_{1}=\left\{n \mid z_{n} \in H_{k^{*}}\right\}$ and $N_{2}=\{n \mid$ $\left.z_{n} \notin H_{k^{*}}\right\}$. If the former is infinite, then evidently the sequence $\left(z_{n}^{\prime}\right)_{n \in N_{1}}$ converges to $z^{\prime}$. Now assume the latter is infinite.

For each $n \in N_{2}$, there is a unique point $y_{n}$ of $H_{k^{*}}$ so that the component of $X \backslash H_{k^{*}}$ containing $z_{n}$ attaches at $y_{n}$. If $N$ is a connected neighbourhood of $z$ containing $z_{n}$, then, since $y_{n}$ is in every arc in $X$ joining $z_{n}$ and $z$ and $N$ contains such an arc, it follows that $y_{n}$ is in $N$. Therefore, the sequence $\left(y_{n}\right)$ converges to $z$. Thus, $\left(y_{n}^{\prime}\right)$ converges in $H_{k^{*}}^{\prime}$ to $z^{\prime}$.

For sufficiently large $n, y_{n}^{\prime}$ is within $\varepsilon$ of $z^{\prime}$. Partition $N_{2}$ into $N_{3}=\left\{n \in N_{2} \mid y_{n}=z\right\}$ and $N_{4}=\left\{n \in N_{2} \mid y_{n} \neq z\right\}$. If the latter is infinite, then choose $n$ sufficiently large so that the $\Delta_{j}$ that attaches at $y_{n}^{\prime}$ has diameter less than $\varepsilon$. Thus, for $n$ sufficiently large and $n \in N_{4}, z_{n}^{\prime}$ is within distance $2 \varepsilon$ of $z^{\prime}$.

Finally, suppose $N_{3}$ is infinite and consider $n \in N_{3}$. Since $z_{n} \notin H_{k^{*}}$, and $y_{n}=z$, it follows that, for $n$ sufficiently large, $z_{n}^{\prime} \in \Delta_{k^{*}}$ and, therefore, $z_{n}^{\prime}$ is within distance $\varepsilon$ of $z^{\prime}$. 


\section{Conclusion}

Generalized thumbtacks may well provide further insight into embeddability into higher genus surfaces. Thomassen [4] proves that if a 2-connected, locally 2-connected compact metric space $M$ does not contain the infinite complete graph as a subspace, then there is a unique compact surface $M^{\prime}$ so that $M$ is homeomorphic to a subspace of $M^{\prime}$ and the set of finite graphs that embed in $M$ is the same as the set of finite graphs that embed in $M^{\prime}$. The locally 2-connected condition is very strong and was introduced to exclude spaces obtained by identifying two (or more) points in a locally connected space. For example, if $x$ and $y$ are distinct points of the sphere, then the space $\Sigma$ obtained by identifying $x$ and $y$ is not a subspace of any compact surface.

We remark that the space $\Sigma$ contains a thumbtack as a subspace. It is natural to wonder about the following.

Conjecture 5.1 Let $X$ be a connected, compact, locally connected metric space not containing a generalized thumbtack. If $X$ contains none of the (finitely many) minimal graphs that do not embed in a given compact surface $\Sigma$, then $X$ embeds in a surface with genus at most that of $\Sigma$.

More weakly, one might conjecture that if the genera of the finite graphs contained in $X$ is bounded, then $X$ embeds in some compact surface.

A necessary condition for a space $X$ to embed in a 2-manifold is that every point have a neighbourhood that embeds in the plane. For this to fail, every neighbourhood of some point (of a compact, locally connected metric space) must contain a generalized thumbtack or a $K_{5}$ or a $K_{3,3}$.

Even if we exclude all generalized thumbtacks, there are several ways a point can have no planar neighbourhood. Here are some natural examples:

- a sequence of disjoint $K_{3,3}$ 's converging to $x$;

- a sequence of $K_{3,3}$ 's converging to $x$ and having only $x$ in common; and

- a sequence of $K_{3,3}$ 's converging to $x$ that are disjoint except that there is one edge of each that properly contains an edge of the next (and these edges are nested).

Is there a finite list $\mathfrak{L}$ of structures, centred on a point $x$, so that a point $x$ of a compact, locally connected metric space has no planar neighbourhood if and only if $x$ is the centre of a structure in $\mathfrak{L}$ ?

Finally, we note that one does not need to exclude infinitely many different subspaces from $X$ to ensure planarity. By considering a subsequence of the simple closed curves making up the web, we can assume the overlapping bridges are either all skew or all 
3-equivalent. If the overlaps are all skew, the subweb is a unique cubic graph (see Figure 1). If they are all 3-equivalent, then for each simple closed curve either all the vertices have degree 4 , producing one transition, or some vertices have degree 3, giving rise to three additional transitions (see Figure 2). Now we can find a subweb so that all of the transitions are of the same type. Thus, there are only five further obstructions to planarity.

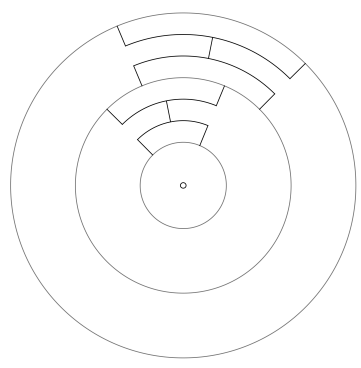

Figure 1: The thumbtack arising from skew transitions.
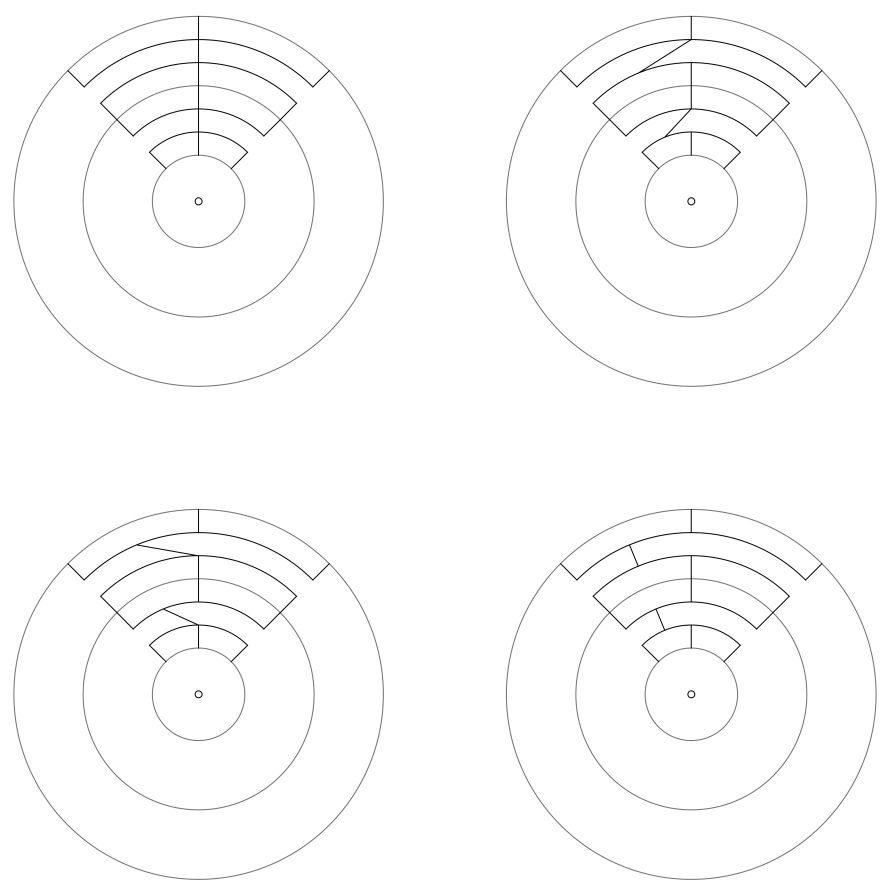

Figure 2: The four thumbtacks arising from 3-equivalent transitions.

\section{References}

[1] K. Kuratowski, Sur le problème des courbes gauches en topologie, Fund. Math. 15 (1930), 271-283. 
[2] R.B. Richter and C. Thomassen, 3-connected planar spaces uniquely embed in the sphere, Trans. Amer. Math. Soc. 354 (2002), no. 11, 4585-4595.

[3] C. Thomassen, The locally connected compact metric spaces embeddable in the plane, Combinatorica 24 (2004), no. 4, 699-718.

[4] C. Thomassen, Classification of locally 2-connected compact metric spaces, Combinatorica 25 (2005), no. 1, 85-103.

[5] W.T. Tutte, Connectivity in Graphs, Oxford University Press, London 1966.

[6] W.T. Tutte, Graph Theory, Cambridge University Press, Cambridge, 2001.

[7] H.--J. Voss, Cycles and Bridges in Graphs, Kluwer, Dordrecht, 1991.

[8] G.T. Whyburn, Cut points in general topological spaces, Proc. Nat. Acad. Sci. U.S.A. 61 (1968), 380-387. 